\title{
Cardiac Endoscopy Enhanced by Dynamic Organ Modeling for Minimally-Invasive Surgery Guidance
}

\author{
Stanislaw Szpala ${ }^{1}$, Gerard Guiraudon ${ }^{2,3,4}$, and Terry Peters ${ }^{1,2,3}$ \\ ${ }^{1}$ Robarts Research Institute \\ ${ }^{2}$ Lawson Health Research Institute \\ ${ }^{3}$ University of Western Ontario \\ ${ }^{4}$ Canadian Surgical Technologies and Robotics (C-STAR) \\ London, Ontario, Canada \\ \{sszpala, tpeters\} @imaging.robarts.ca
}

\begin{abstract}
The development of a 3D image guidance environment will significantly enhance the performance of minimally invasive robotically assisted cardiac surgery. We have previously reported early progress on generation of such virtual environment, and linking it with traditional modalities like endoscopy. This paper discusses one aspect of the surgical navigation system: near-real time animation of the model of the beating heart phantom based on CT volumetric images, and overlaying the virtual view with optical images from a tracked endoscope. We have demonstrated that in the plane of the endoscopic image, normal to the endoscope optical axis, and intersecting the heart surface, the real and virtual endoscopic images can be registered to within $-2.6 \mathrm{~mm}$ and $+0.8 \mathrm{~mm}$ depending on the phase of the cardiac cycle, and with almost negligible time lag between the images.
\end{abstract}

\section{Introduction}

In 1999 Loulmet et al. [1] and Mohr et al. [2] independently reported totally endoscopic coronary artery bypass graft (CABG) performed on humans, thus initiating the application of minimally invasive techniques to cardiac surgery. The procedure was successful in part due to development of surgical telemanipulation systems, like da Vinci (Intuitive Surgical Inc., Sunnyvale, CA) and Zeus (Computer Motion Inc., Goleta, CA), providing improved control over surgical instruments. Further development of the procedure is hampered by the lack of image (CT or MR) guidance during both the surgery planning and the actual procedure. However, a virtual environment based on pre- and intra-operative images can complement the limited field of view of an endoscope, the primary intra-operative observational tool, and its inability of viewing visually obstructed tissue.

We have been developing a 3D image guidance environment for minimally invasive and robotically assisted CABG, with various pre- and intra-operative imaging modalities combined to create virtual cardiac surgical platform. Preliminary developments were published by Chiu et al. [3], who demonstrated superposition of endo-

R.E. Ellis and T.M. Peters (Eds.): MICCAI 2003, LNCS 2878, pp. 499-506, 2003.

(C) Springer-Verlag Berlin Heidelberg 2003 
scopic images with CT-reconstructed thorax phantom, and Lehmann et al. [4],[5], who incorporated virtual animated coronary arteries derived from 2D bi-plane angiograms. Recently, we demonstrated the fusion of tracked endoscope images with CTderived models in neurosurgical context [6], while Shahidi et al. [7] reported clinical applications of this approach. Mourguess and Coste-Manière reported an overlay of endoscopic and CT-generated images of a plastic (non-beating) heart phantom based on the location of a robotic arm supporting the endoscope [8].

While previous work has dealt with static-image fusion, in this paper we emphasize the ability to fuse real and virtual images that are dynamically changing due to the beating of the heart. We demonstrate the dynamic fusion of optical endoscopic images of a beating heart phantom with the corresponding animated virtual images generated from a sequence of 3D-CT volumes, acquired from a gated multi-slice helical scanner. The overlay is accurate not only with respect to the arbitrary pose of the (tracked) endoscope, but also the phase of the "cardiac cycle" of the phantom. We quantify the errors of the overlay in terms of the time lag and spatial discrepancy.

\section{Methods}

\subsection{General Description of the System}

In order to simulate an environment similar to the heart beating inside the human thorax, we used a beating heart phantom. The phantom was visualized with a tracked endoscope (Figure 1). The dynamic virtual heart model was constructed from 3D-CT images collected during a "cardiac cycle". Fourteen such images were used as a database from which individual images (representing separate phases of the heart) were retrieved and displayed together with the optical endoscopic view. Overlay of the optical and virtual images was accomplished through placing the segmented CT-scan of the heart phantom in virtual space behind the "semi-transparent plane" on which the endoscope image was displayed. To allow matching of the $2 \mathrm{D}$ endoscope view with the $3 \mathrm{D}$ image of the phantom, the rendering camera was placed at the location in the virtual scene corresponding to the focal

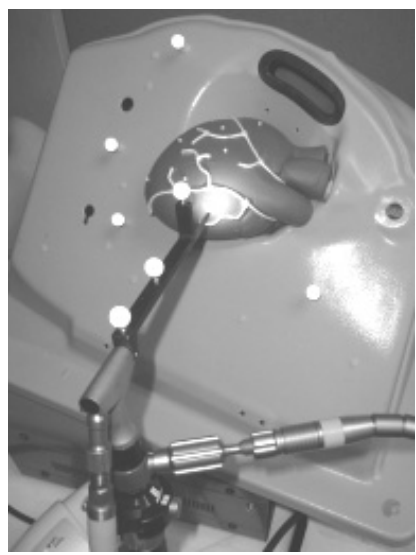

Fig. 1. Optically tracked endoscope pointing at the tracked heart phantom. point of the endoscope lens. The location of the focal point with respect to the heart phantom was established using POLARIS tracking system (NDI, Waterloo, ON, Canada). The pose of the virtual model was continuously updated to account for the relocation of the endoscope and pulsation of the heart phantom. 


\subsection{Components}

A pulsatile (inflating/deflating) heart phantom (Limbs \& Things Limited, Bristol, UK, model 40503) was used together with the supplied air pump / controller assembly, which adjusts the phantom volume. The controller was modified to provide an " $\mathrm{r}$ wave" equivalent phase synchronization signal (generated on a preselected phase of the air pump), which is fed to the computer via the parallel port. A homing procedure was added to correlate the pressure in the phantom (controlling the phase of the pulsation) with the position of the piston in the air pump. During the homing procedure, the piston in the air pump was allowed to move until detection of the rise of the synchronization signal. The static pressure corresponding to phase zero (maximum volume) was adjusted to $15 \pm 1 \mathrm{mmHg}$ as measured with a digital blood pressure monitor.

Coronary vessels were simulated using insulated copper wires attached to the phantom with silicone. For validation purposes, $2 \mathrm{~mm}$ in diameter white-painted ball-bearings were glued to the phantom.

The 3D-CT data for the virtual heart phantom was collected with a GE Medical System LightSpeed helical multi-slice CT scanner (8 slices, $1.25 \mathrm{~mm}$ thick each, $0.5 \mathrm{~s}$ rotational period, $120 \mathrm{kV} / 300 \mathrm{~mA}$ ). Due to limitations of the image reconstruction software, only 7 phases (each lasting $7 \%$ of the heart period) were reconstructed out of the first $49 \%$ of the period. The following 7 phases were assumed to be identical to the corresponding reconstructed phases assuming cosine time dependence (following phase sequence: 12345677654321 ). As the reconstructed data covered $49 \%$ instead of $50 \%$ of the period, the 8 -th phase was set to start at $51 \%$ of the period. The consequences of this incomplete reconstruction (missing $2 \%$ ) are discussed later.

Tracking of the position and orientation of both the endoscope and the heart phantom was performed with a POLARIS optical tracking system. For endoscope tracking, a custom-made tracking probe consisting of three retroreflective balls was mounted onto the endoscope, while for the heart phantom tracking, another set of three such balls was attached rigidly to the phantom base. As the heart phantom may be placed at the base in a repeatable manner, tracking the base was sufficient to establish the pose of the phantom.

An Aesculap (Melsungen, Germany) $0^{0}$ fixed-focus neurosurgical endoscope was used for this study. The lens barrel distortion was removed at the software level using a technique similar to the one described by Shahidi et al. [7].

\subsection{Software}

The software controlling the system was written in Python 2.1 using VTK 4.1 (www.vtk.org) and Atamai 1.0 (www.Atamai.com) classes. The software was developed under Windows 2000, and executed on an AMD Athlon MP 1800+ machine with ASUS V8200 Series v31.40b display adapter and Matrox Corona video digitizer used to digitize the endoscope images. The program allows display of semitransparent endoscope view of the heart phantom overlaid with a view constructed 
from the same pose of the virtual equivalent of the phantom. The transparency fraction is user-adjustable for optimum visualization.

To ensure that the real and virtual views from the arbitrarily placed endoscope are properly overlaid, the pose matrices are read from the tracking devices attached to the endoscope and the heart phantom independently, and subtracted to obtain the pose matrix of the endoscope with respect to the phantom. The pose matrices are updated every $20 \mathrm{~ms}$, and averaged when the endoscope is not moving (to remove jitter in the coordinates being returned by the POLARIS system).

The rendering camera is placed in the $3 \mathrm{D}$ virtual scene at the location corresponding to the focal point of the endoscope lens. A rectangular plane displaying a scaled endoscope image (updated at $14 \mathrm{~Hz}$ ) is placed normal to the endoscope optical axis in front of the rendering camera at arbitrarily selected distance of $15 \mathrm{~mm}$. The endoscope image is radially distorted according to the previously determined quadratic barrel-distortion correction. This deformation is performed in real time without additional software overhead using texture mapping capabilities of the graphic card. Isosurfaces generated from the preoperative 3D-CT scans using the marching cubes algorithm are used for the virtual version of the heart phantom.

The isosurfaces are transformed from 3D pixel coordinates of the CT scan to 3D real-space coordinates (in $\mathrm{mm}$ ). 14 such isosurfaces (see section 2.2) constitute the dynamic volumetric image throughout the cardiac cycle, with selection of individual volume being governed by the amount of elapsed time since detection of the previous pseudo-ECG trigger.

\section{Results}

Evaluation of the system consists of both static and the dynamic cases. The former pertains to the non-beating heart phantom, while the latter to the beating phantom.

\subsection{Static}

Static overlay of the real (optical endoscope) and virtual images of the heart phantom is demonstrated in Figure 2 (left). The view as seen with the endoscope is shown in the center, while the corresponding virtual view is shown on the right. The opacity of the endoscopic view in the overlaid image was set to $75 \%$ allowing simultaneous viewing of the real and the virtual images.

To ensure reproducibility of the base-line (static) measurements, static registration errors were determined using fiducial markers (steel ball-bearings) mounted on a rigid wooden model of the heart instead of the flexible heart phantom. The true 3D coordinates of the markers were derived from a separate 3D-CT scan. Since we only have a $2 \mathrm{D}$ view of the scene, it is impractical to calculate the true target registration errors (TRE), i.e. the distance between a point in 3D space and its equivalent in "endoscope space". Instead, we calculated a 2D equivalent of TRE, defined as the vector between the investigated point in real space and its image in the endoscope plane, af- 
ter conceptually shifting the plane along the endoscope axis to intercept the investigated point. This is referred to from now on as "projected error vector". The length of the projected error vector is expressed in millimetres, and serves as an estimate of the projected localization of an organ in real space based on its endoscope image overlaid with the virtual equivalent of that organ.

The projected errors were examined for variety of endoscope poses with respect to the static heart phantom. The average absolute value of the projected error for 100 data points was $\left\langle\left|\mathrm{e}_{\mathrm{p}}\right|>=0.50 \mathrm{~mm}\right.$, while its standard deviation, $\sigma=0.33 \mathrm{~mm}$.
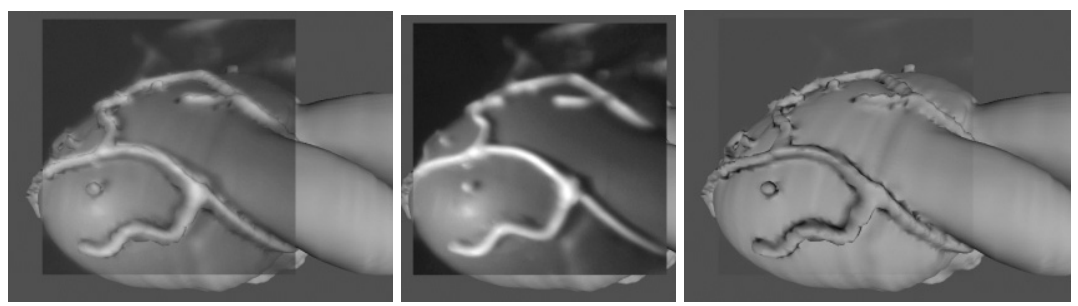

Fig. 2. Static overlay (left) of the endoscopic (center) and the virtual (right) heart phantom at $75 \%$ opacity of the endoscopic image.

\subsection{Real-Time Acquisition for Pulsating Heart}

To verify the overlap of the optical and virtual images of the pulsating heart phantom, snapshots of the rendering window were saved and analyzed for 14 phases of the phantom pulsation. To avoid the influence of the computer-time consuming windowsaving procedure $(\sim 0.2 \mathrm{~s})$ on the display of the subsequent scheduled heart phase, the snapshot corresponding to the next phase of pulsation was taken after receiving the next ECG-like synchronization signal (triggering the heart-display cycle). Examples of the snapshots collected at the heart rate of $42 \mathrm{bpm}$ are shown in Figure 3. Two phases are displayed: upon receiving the ECG signal, which corresponds to the maximum volume of the phantom (left) and $0.53 \mathrm{~s}$ later (intermediate phase) (right). The opacity of the endoscopic view (displayed as a square) was set to $65 \%$ to allow simultaneous viewing of the optical and virtual images.
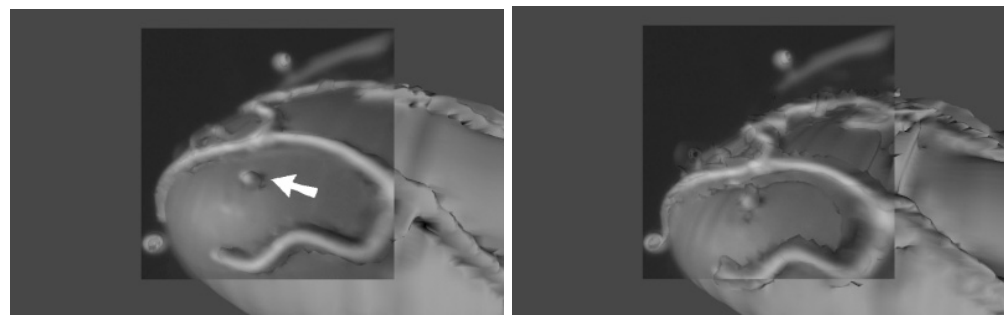

Fig. 3. Snapshots collected with the heart phantom beating at $42 \mathrm{bpm}$ : at the beginning of the cycle (left) and $0.53 \mathrm{~s}$ later (right). The smearing of the virtual features in the latter is caused by the deflating motion of the phantom during the CT scan. The arrow indicates the fiducial used in the error analysis. 
In all cases, visually satisfactory overlap of the optical and virtual images is evident, although smearing (caused by movement of the phantom during the CT scan) of the virtual image is visible in the latter image. The smearing increases with the increase of the speed of deflation / inflation of the phantom, and is smallest when the volume of the phantom is at the minimum or the maximum. The errors in time synchronization and the spatial shift between the optical and virtual images were determined based on the screen coordinates of a fiducial marker placed on the surface of the phantom, see Figure 3. Similarly to static validation, the screen-pixel coordinates of the fiducials were converted to projected error vectors (in XY plane).

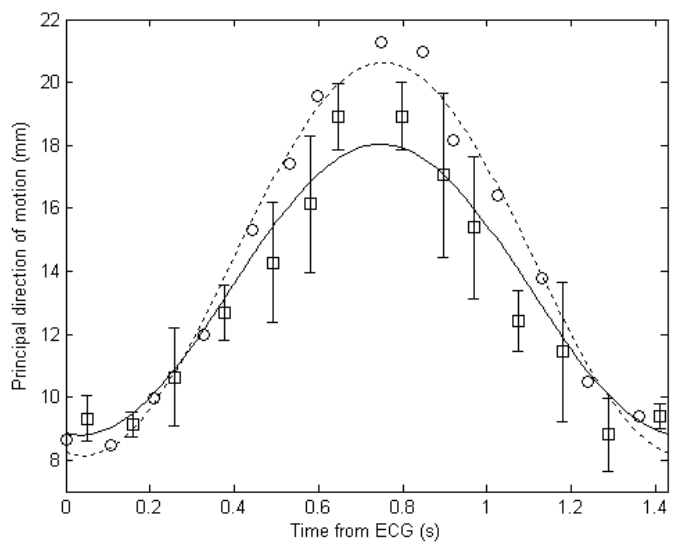

Fig. 4. Time dependence of the position (along the principal direction of motion) of the optical (circles) and virtual (squares) fiducial marked with an arrow in Fig. 3. The analytical fits are also plotted.

The overlap of the optical and virtual projected error vectors was analyzed with respect to the time elapsed from the cycle start, see Figure 4. The projected error vectors of both optical and virtual fiducial are expressed in terms of the coordinate along the principal direction of oscillation of the optical fiducial (considered as the reference). Each virtual data point was shifted right by $0.05 \mathrm{~s}$ to display the points at the average viewing instant, rather than when the program started displaying the corresponding virtual image. Large error bars surrounding the virtual data points originate from smearing of the virtual features due to the deflating and inflating motion of the phantom during the CT scan, see Figure 3(right). Cosine functions with the period $\mathrm{T}=$ $1.428 \mathrm{~s}$ (corresponding to the heart rate of $42 \mathrm{bpm}$ ) were fitted independently to the optical and the virtual data points (see Table 1 for fitted parameters):

$$
\mathrm{y}=A \cos \left(2 \pi / \mathrm{T}\left(\mathrm{t}-t_{0}\right)\right)+B
$$

Table 1. Fitted parameters of the cosine function (equation 1) modeling the time oscillation of the optical and the virtual fiducial along the principal coordinate of the oscillation.

\begin{tabular}{|c|c|c|c|}
\hline & $\boldsymbol{A}(\mathbf{m m})$ & $\boldsymbol{t}_{0}(\mathbf{s})$ & $\boldsymbol{B}(\mathbf{m m})$ \\
\hline Optical & $-6.2 \pm 0.5$ & $0.04 \pm 0.02$ & $14.4 \pm 0.4$ \\
\hline Virtual & $-4.6 \pm 0.8$ & $0.03 \pm 0.04$ & $13.4 \pm 0.7$ \\
\hline
\end{tabular}

Since the time error $t_{0}$ between the optical and the virtual cosines is small compared to the interval between consecutive heart phases of $0.11 \mathrm{~s}$, the display of both the optical and the virtual heart phases exhibit a negligible delay from the ECG synchronization signal. The phase difference between the two cosines is $0.01 \pm 0.05 \mathrm{~s}$, and denotes the error in time synchronization between the endoscopic and virtual images. 
As the synchronization error constitutes barely $0.7 \%$ of the motion period, the time synchronization between the optical and virtual images can be considered satisfactory. Although the absolute value of the difference in the amplitude shift $B$ between the optical and the virtual cosines is small compared to the amplitude $A(1 \mathrm{~mm}<<$ $6.2 \mathrm{~mm}$ ), there is a considerable discrepancy between the amplitudes. The amplitude $A$ of the virtual cosine is smaller by $25 \%$ with respect to the amplitude of the optical cosine. The amplitude $A$ combined with the shift $B$ represent a shift between the two cosines of $0.8 \mathrm{~mm}$ for the maximum volume of the phantom, and $-2.6 \mathrm{~mm}$ for the minimum volume. This is partially due to limitations of the reconstruction of the virtual images from the helical scan: each image is the average of $7 \%$ of the cycle, and averaging around the minimum or the maximum values of the curve "flattens-out" the peaks. Also the images were not reconstructed between $49 \%$ and $51 \%$ of the cycle, resulting in additional "flattening" of the curve around $0.75 \mathrm{~s}$ from the beginning of the cycle.

\section{Discussion}

This work is preliminary and represents only the first steps towards the goal of achieving matched dynamic endoscope and model images in a surgical context. Although the virtual images are well time-synchronized with the optical images, the spatial discrepancy is noticeable. As explained in section 3.2, the discrepancy is caused by limitations of the dynamic volumetric data extracted from the CT scan. While time-frozen static images of the phantom corresponding to each dynamic "snapshot" could have been collected, this situation ignores the limitations imposed by a real beating heart. Instead, we plan to warp the pseudo-static 3D model of the heart of the operated patient (acquired during diastole) to represent the various images at different phases of the cardiac cycle by using angiogram data acquired in the operating room [5].

In a clinical situation we plan to track the heart within the thorax using intraoperative angiogram data, as well as employing tracked trans-esophageal ultrasound, combined with image-based registration proposed by Mori et al.[9].

The cardiac phase is currently estimated based on the time elapsed from the last ECG-like signal, and the procedure suffers from irregularity of the heart rate. For clinical applications, the phase may be determined from analysis of the shape of the ECG signal.

This system displays 14 images per second, a limitation imposed by the currently available speed of the graphics, and the complexity of the rendered virtual model.

The phantom motion (inflating/deflating movement following a cosine time dependence) is dictated by the characteristics of the commercially available heart/thorax phantom we are using, and does not reflect accurately the dynamics of a real heart. The next phase of this work will involve validation using an in-vivo porcine model.

Acknowledgments. The authors would like to thank D. Gobbi, R. Gupta, M. Wierzbicki, A. So, M. Drangova, C. Norley and C. Nikolov for assistance. This work was 
supported by grant NA4755 from the Ontario Heart and Stroke Foundation, R3146A02 from National Science and Engineering Research Council of Canada, and Ontario Consortium for Image-guided Surgery and Therapy.

\section{References}

1. D. Loulmet, A. Carpentier, N. d'Attellis, A. Berrebi, C. Cardon, O. Ponzio, B. Aupecle, JYM. Relland, "Endoscopic coronary artery bypass grafting with the aid of robotic assisted instruments", J Thorac Cardiovasc Surg 118(1), 4-10 (1999).

2. F.W. Mohr, V. Falk, A. Diegeler, R. Autschbach, "Computer-enhanced coronary artery bypass surgery", J Thorac Cardiovasc Surg 117(6), 1212-1214 (1999).

3. A.M. Chiu, D. Dey, M. Drangova, W.D. Boyd, TM. Peters, "3D-image guidance for minimally invasive robotic coronary artery bypass”, Heart Surg Forum 2000-9732(3), p. 224-231 (2000).

4. G. Lehmann, A. Chiu, D. Gobbi, Y. Starveld, D. Boyd, M. Drangova, TM. Peters, "Towards dynamic planning of minimally invasive robotic cardiac bypass surgical procedures", MICCAI 2001: LNCS 2208, W. Nieslen and M. Viergover (eds), 368-375.

5. G. Lehmann, D. Habets, D.W. Holdsworth, TM. Peters, M. Drangova, "Simulation of intraoperative 3D coronary angiography for enhanced minimally invasive robotic cardiac intervention", MICCAI 2002(2): LNCS 2489, T. Dohi and R. Kikinis (eds), 268-275.

6. D. Dey, D.G. Gobbi, P.J. Slomka, K.J.M. Surry, TM. Peters, "Automatic fusion of freehand endoscopic brain images to three-dimensional surfaces: creating stereoscopic panoramas", IEEE Trans Med Imaging 21(1), 23-30 (2002).

7. R. Shahidi, M.R. Bax, C.R. Maurer, J.A. Johnson, E.P. Wilkinson, B. Wang, J.B. West, M.J. Citardi, K. H. Manwaring, R. Khadem, "Implementation, calibration and accuracy testing of an image-enhanced endoscopy system”, IEEE Trans Med Imaging 21(12), 1524-1535 (2002).

8. F. Mourgues and È. Coste-Manière, "Flexible calibration of actuated stereoscopic endoscope for overlay in robot assisted surgery", MICCAI 2002(1): LNCS 2488, T. Dohi and R. Kikinis (eds), 25-34.

9. K. Mori, D. Deguchi, J. Sugiyama, Y. Suenaga, J. Toriwaki, CR. Maurer, H. Takabatake, H. Natori, "Tracking of a bronchoscope using epipolar geometry analysis and intensitybased image registration of real and virtual endoscopic images, Med Image Anal 6(3), 321-36 (2002). 